AperTO - Archivio Istituzionale Open Access dell'Università di Torino

\title{
Comparing different methods to retrieve cloud top height from Meteosat satellite data
}

\section{This is the author's manuscript}

Original Citation:

\section{Availability:}

This version is available http://hdl.handle.net/2318/1653505

since 2017-11-29T16:32:11Z

Publisher:

SPIE

Published version:

DOI:10.1117/12.2195012

Terms of use:

Open Access

Anyone can freely access the full text of works made available as "Open Access". Works made available under a Creative Commons license can be used according to the terms and conditions of said license. Use of all other works requires consent of the right holder (author or publisher) if not exempted from copyright protection by the applicable law. 


\title{
Comparing different methods to retrieve cloud top height from Meteosat satellite data
}

\author{
Tabone I. ${ }^{a}$, Briz S. ${ }^{b}$, Anzalone A. ${ }^{c}$, De Castro A.J. ${ }^{b}$, Lopez F. ${ }^{b}$, Ferrarese S. ${ }^{a}$, Isgrò F. ${ }^{d}$, \\ Cassardo C. ${ }^{a}$, Cremonini R. ${ }^{a}$, Bertaina M. ${ }^{a}$ \\ ${ }^{a}$ Dipartimento di Fisica, Università degli Studi di Torino and INFN - Sezione di Torino, via P. \\ Giuria 1,Torino, Italy; \\ ${ }^{b}$ Universidad Carlos III de Madrid, Avda. de la Universidad 30, 28911 Leganés, Spain; \\ ${ }^{c}$ INAF - IASF, Istituto di Astrofisica Spaziale e Fisica Cosmica, Palermo via U.La Malfa 153, \\ Palermo, Italy and INFN - Sezione di Catania, Italy; \\ ${ }^{d}$ DIETI, Università degli Studi di Napoli Federico II, via Claudio 21, Napoli, Italy and INFN \\ - Sezione di Napoli, Italy
}

\begin{abstract}
Cloud parameters such as the Cloud Top Height (CTH), Cloud Top Temperature (CTT), emissivity, particle size and optical depth have always been matter of interest for the atmospheric community. Particularly the $\mathrm{CTH}$ provides information leading to better understand the cloud radiative effects. Although there are many metereological satellites providing the $\mathrm{CTH}$, there are other sensors, not devoted to this purpose, that give some information from which this crucial parameter can be estimated. In this contribution we will describe three different methodologies to retrieve the CTH. The first technique is based on stereo-vision algorithms and requires two different views of the same scene and does not need of extra atmospheric information. In the second one, brightness temperatures in two IR spectral bands are converted to real cloud temperature by means of the proposed algorithms. From the CTT, the CTH is estimated using temperature vertical profiles (measured or modeled). The third technique retrieves the $\mathrm{CTH}$ from the output parameters of post event simulations performed by a Numerical Wheather Prediction (NWP) model that in this work will be the mesoscale model WRF (Weather Research Forecast). This article presents a preliminary work, in which the heights retrieved by the three methodologies applied to the geostationary satellite Meteosat 10 are compared with the heights given by MODIS sensor installed on the polar satellite AQUA. These promising results show that valuable information about CTH can be retrieved from Meteosat which provides high frequency and large scale data useful for weather and climate research.
\end{abstract}

Keywords: Split-Window-Algorithm, Stereo-Vision-Algorithm, WRF model, Meteosat, Infrared, Cloud-TopHeight

\section{INTRODUCTION}

Clouds play a fundamental role in weather and climate due to their interaction with shortwave solar and longwave thermal radiation. The radiative processes are sensitive to cloud parameters such as the Cloud Top Height (CTH), Cloud Top Temperature (CTT), cloud emissivity, cloud particle size and cloud optical depth. Thus, the monitoring of cloud parameters has been subject of interest for the atmospheric science community. In particular, CTH studies provide information on cloud vertical structure leading to better understand the cloud radiative effects.

Commonly, meteorological satellites provide the CTH. However, other spaceborn sensors with narrower spectral range offer the corresponding information from which this crucial parameter can be estimated. The objective of this work is to introduce an approach, which combines advantages of three conventional methodologies to retrieve

Further author information: (Send correspondence to Susana Briz, Anna Anzalone)

Susana Briz: E-mail: sbriz@fis.uc3m.es

Anna Anzalone: E-mail: anna.anzalone@ifc.inaf.it

Remote Sensing of Clouds and the Atmosphere XX, edited by Adolfo Comerón, Evgueni I. Kassianov, Klaus Schäfer, Richard H. Picard, Konradin Weber, Proc. of SPIE Vol. 9640 , 96400C

2015 SPIE · CCC code: $0277-786$ X/15/\$18 - doi: 10.1117/12.2195012

Proc. of SPIE Vol. $964096400 \mathrm{C}-1$ 
the CTH. This approach utilizes data collected by geostationary Meteosat Second Generation (MSG) satellites (two infrared channels). To demonstrate the potential of this approach, the MSG-based CTH estimations are compared with those obtained from quasi-simultaneous Moderate Resolution Imaging Spectroradiometer (MODIS) data.

The first method is based on stereo vision algorithms and requires two different views of the same scene. Since the stereo-based CTH reconstruction relies on image analysis, it does not need the atmospheric information. The second method is based on the brightness temperatures in infrared (IR) spectral region estimated from space and complementary temperature vertical profiles. These profiles can be provided by infrequent radiosound measurements or post-event simulations performed by Numerical Wheather Prediction (NWP) models, including the mesoscale Weather Research Forecast (WRF) model. The third method utilizes explicitly the outputs of the WRF model to estimate the CTH. These outputs define the atmospheric state of the scene observed by the MSG satellites.

Here, three methodologies outlined above are applied to data collected by the two MSG satellites (Meteosat 9 and 10) with high temporal resolution over large areas. The collected data represent quasi-synchronous images of the same scene from two different points of view for stereo vison analysis. Moreover, the collected data provide the bi-spectral information required for radiative transfer calculations.

This work is structured as following. Three methodologies are explained in section 2. Section 3 describes the collected data. The results are discussed in section 5 and the main conclusions are summarized in section 6 .

\section{METHODS}

\subsection{Stereo Method}

Thanks to the satellite technological progress, it is possible to retrieve the CTH with the stereo approach that depends only on an accurate image analysis and on the geometry of the system, without using information of vertical temperature profiles or other atmosphere parameters. The basis of this method consits in exploiting 2 -D images of the same object acquired by different view angles and the parallax effect to reconstruct the 3-D structure.

In remote sensing it can be accomplished configuring the satellites as stereo systems, that means considering images provided by couple of sensors with overlapped field of views and located at different positions as can be done with the geostationary satellites. Or also stereo pair images from instruments designed for this purpose and aboard polar satellites. In this work we have considered the first option.

\subsubsection{Stereo Parameters}

Two elements are fundamental for the application of the stereo method: the distance between the two sensors called baseline and the time delay between two acquisitions of a scene. From the baseline depends the range of cloud heights that can be detected, while the time lag garantees that other factors as the cloud movement does not affect the final results. Of course many other elements need to be considered such as the similarity of both devices, pixel resolution, satellite position data.

In our case we have applied the method to the combination of the Meteosat Second Generation satellites 9 (MSG2) and 10 (MSG-3), as a compromise between the previous characteristics. They are located on the Equatorial plane respectively at $0^{\circ}$ and $9.5^{\circ} \mathrm{E}$ at about $36,000 \mathrm{~km}$ of altitude. MSG-3 scans the whole Europe and Africa every 15 minutes, while the Rapid Scan System of MSG-2 provides data for the Northern Hemisphere every 5 minutes. In this way two different views for the same area with a pixel resolution at the Sub Satellite Point of $3 \mathrm{~km}$, are obtained. We have chosen this coupling due to the same SEVIRI instruments aboard the orbiters and for the quasi-simultaneous acquisitions. Unfortunately the short baseline between the two positions $\approx 7018 \mathrm{~km}$, respect to the Meteosat altitude, is not enough to provide an accurate reconstruction of the height. Therefore it is expected that the whole range of heights of clouds present in the scene, will not be well reconstructed. Following ${ }^{1}$ the accuracy of the $\mathrm{CTH}\left(\sigma_{C T H}\right)$ estimated from a stereo system can be expressed as

$$
\sigma_{C T H}=\frac{\sigma_{d}}{b / H_{s}}
$$


where $\sigma_{d}$ is the disparity estimation error on ground, $b$ is the baseline of the stereo system, i.e. the distance in space between the two observations, and $H_{s}$ is the height of the satellite. Using Equation 1 and considering an average disparity of no more than half a pixel we have a $\sigma_{d}= \pm 1.5 \mathrm{~km}$, obtaining an accuracy within $7.6 \mathrm{~km}$. Hence if the disparity is estimated with an accuracy of half pixel, in this configuration clouds with heights $h \in[7.5,15] \mathrm{km}$ may not be distinguished, while lower clouds will be merged to the background. The two imaging devices are quasi-synchronous, and ancillary data include also the actual satellite positions for each row of the images that will be utilised for the CTH estimation.

It is worth pointing out that this measure of the accuracy considers only the geometry of the system, and does not take into account other sources of apparent motion, as the wind.

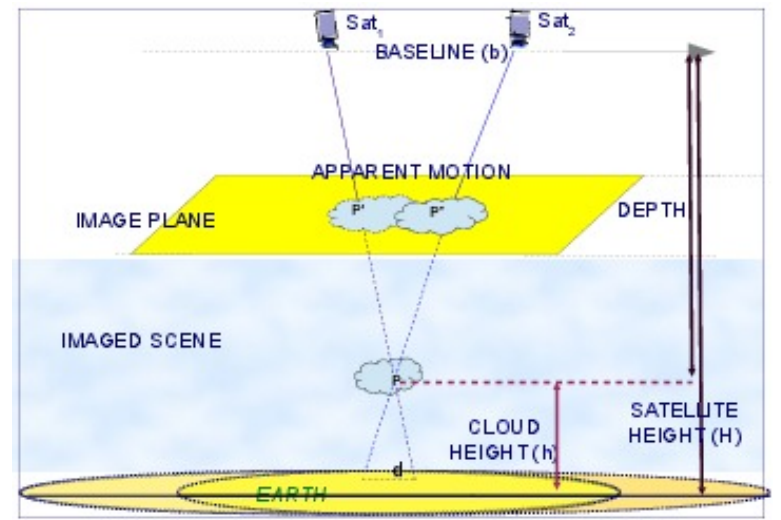

Figure 1: Stereo Reconstruction. The scheme shows the height reconstruction for the cloudy pixel $P$. Its projection points $\left(P^{\prime}\right.$ and $\left.P^{\prime \prime}\right)$ are detected by the matching step and the depth is recovered by triangulation. Finally the height is calculated by subtracting these values from the satellite altitude.

In Fig.1 it is visible the parallax effect as an apparent motion of the cloud, shown in the overlapped part of the two views.

\subsubsection{Stereo Algorithm}

The main task of stereo retrieval is the matching step that identifies the same features in the two views and estimates for each pixel the parallax effect highlighted as an apparent motion of the point and named as disparity. The final reconstruction is then accomplished by triangulating the reconstructed optical rays (Fig.1). Different algorithms are present in literature and for this work we have tested a modified version of the algorithm in. ${ }^{2}$ It is based on the idea that the height can be estimated working on a coarser resolution than the single pixel, segmenting the image in slices of pixels, corresponding to slices of the atmosphere, having values inside fixed ranges. This procedure gives rise to a set of regions (binary masks) in both images that will be matched to assign them a disparity. Finally the so estimated disparity is geometrically converted into height and assigned to each pixel by intersecting the optical rays. They are given by the two projections on the Earth, of conjugate points i.e.the successfully matched points and the actual satellite positions at the time of the acquisitions, that are supplied by the database for each row of the images. Due to the uncertainties in the correct orbiter location, to the geolocation precision and the coupling made by the matches, the two optical vectors cross each other at certain distances. Hence the minimum distance is chosen as the final CTH.

\subsection{WRF Cloud Fraction Method}

The CTH can be retrieved also from NWP models primary outputs. In this work the limited area WRF model Version 3.6 has been considered. The WRF model is a NWP model and atmospheric simulation system designed with fully compressible and Euler non-hydrostatic primitive-equations. Horizontal nesting is possible, allowing higher resolutions to be focused over a region of interest. The main WRF parameterizations regard 
micro-physics, cumulus parametrization, surface physics, boundary layer physics, atmospheric radiation physics. Technical details are fully reported in. ${ }^{3}$

Many WRF simulated atmospheric variables can be used to evaluate the height of the clouds. A combination of outputs such as the Cloud Water Mixing Ratio (QCLOUD), Cloud Ice Mixing Ratio (QICE), Pressure and Geopotential is used for example by the WRF post-processing tool VAPOR, an Open Source application developed by the National Center for Atmospheric Research. ${ }^{4}$ In this study a technique exploiting the 3D WRF Cloud Fraction variable is investigated. The cited output describes the fraction of cloudiness in a model grid-box, assuming values from 0 (blue sky box) to 1 (box completely cloudy). The methodology here used is based on this simple procedure:

- fix a Cloud Fraction threshold to name when a box is cloud or not;

- first consider one vertical column of the domain;

- scan level by level (starting from the uppest grid level set in the simulation and going down to the troposphere) the cloud fraction of the grid boxes until the first cloudy grid box is detected. The level at which this occurs is then considered the height of the top of the cloud;

- iterate the procedure for all the vertical columns set in the simulation to get a $2 \mathrm{D}$ matrix of $\mathrm{CTH}$ for the whole simulation domain.

\subsection{Radiative Transfer Methods}

Radiative transfer methods exploit the relation between the cloud temperature and the radiance emitted by the cloud according to the Planck's law. A sensor measuring the radiance emitted by the cloud could retrieve the cloud temperature. Then from vertical profiles of temperature the cloud height can be estimated. However this relation it is not straightforward since the radiance received by the sensor is not the one emitted by the cloud since the atmosphere interacts with that radiation and also the Earth's surface influences if the cloud is not thick enough. For this reason the temperature retrieved by the sensor is not the cloud temperature and some procedures and algorithms are needed to retrieve the cloud temperature. There are many procedures to retrieve the cloud top temperature (CTT) from brightness temperature measurements by SWAs, ${ }^{5,6}$ weighting

functions, ${ }^{7}$ etc. Some of them rely on additional information such as radar, lidar. The idea of this work is to develop independent algorithms to study their feasibility and then to combine them according their capabilities.

\subsubsection{The SWA-RTE Method}

The radiative behaviour of clouds can be described by the Radiative Equation Transfer (RTE) (eq. 2):

$$
L(\nu)=\varepsilon_{s} B\left(T_{s}, \nu\right) \tau_{0}^{c}(\nu)\left(1-\varepsilon_{c}\right) \tau_{c}^{t o p}(\nu)+L_{0}^{c}(\nu)\left(1-\varepsilon_{c}\right) \tau_{c}^{t o p}(\nu)+\varepsilon_{c} B\left(\tau_{c}, \nu\right) \tau_{c}^{t o p}(\nu)+L_{c}^{t o p}(\nu)
$$

Where:

$\mathrm{B}(\mathrm{T}, \nu)$ is the blackbody radiance given by the Planck's law at temperature $\mathrm{T}$ and wavelength $\nu$;

$T_{s}$ and $T_{c}$ are the surface temperature and the cloud top temperature, respectively;

$\varepsilon_{s}$ is the emissivity of the Earth's surface;

$\varepsilon_{c}$ is the emissivity of the cloud;

$\tau_{0}^{c}(\nu)$ is the atmospheric transmittance between the surface and the cloud;

$\tau_{c}^{t o p}(\nu)$ is the atmospheric transmittance between the cloud and the top of the atmosphere;

$L_{0}^{c}(\nu)$ is the radiance of the atmosphere between the surface and cloud;

$L_{c}^{t o p}(\nu)$ is the radiance of the atmosphere between cloud and the sensor.

Considering eq.2, the cloud radiance $B\left(T_{c}, \nu\right)$ could be calculated from the radiance measured by the sensor $L(\nu)$ knowing the atmospheric transmittance and the cloud emissvity. Then the cloud temperature can be calculating by inverting the Planck's law. However it is obvious that for those calculations the height of the cloud should be known and it is precisely the unknown of the problem. A first approximation of eq.2 for high clouds consists of neglecting the atmospheric absorption/emission above the cloud. In this case, the transmittance between the Earth's surface and the cloud, $\tau_{0}^{c}(\nu)$ could be considered as the transmittance of the entire atmosphere 
and the transmittance between the cloud and the sensor could be approximated to 1 . For the same reason the radiance emitted by the atmosphere above the cloud could be neglected $L_{c}^{t o p}(\nu)$ and that one beneath the cloud, $L_{0}^{c}(\nu)$, could be approached to the radiance emitted by the whole atmosphere. In this way the cloud temperature could be retrieved from the brightness temperature calculated with the RTE without knowing the cloud height. Nevertheless calculating the cloud BT from the radiance provided by the sensor requires the emissivity value. However, considering the same equation in the band centered in $12 \mu \mathrm{m}$, both the emissivity and the BT can be retrieved. After that, the cloud temperature can be calculated from the BT in band centered in $10.8 \mu m$, which is less affected by the atmosphere.

The atmospheric radiance and transmittance are calculated for the appropriate atmospheric conditions using MODTRAN code. In addition the radiance emitted by the Earth's surface is also required. For this work, this radiance is calculated from the surface temperature provided by MODIS conveniently adapted to the MSG resolution.

However, as stated above, this approximation only is applicable to high clouds. Instead, for low clouds a SWA has been designed considering specifically the MSG bands. The procedure to calculate the algorithm is the same as the one detailed in ${ }^{8}$ but for MSG bands the best fitting is attained with a second order polinomial in $T_{B 1}$ and $T_{B 2}$.

At this point a criterion to distinguish between low and high clouds must be established. As the starting point is the BT in MSG bands and these values are quite similar, a threshold in the Brigthness Temperature Differences (BTDs) has been conveyed. To set this value MODIS images have been used. Since MODIS products provides a great deal of information (BTs in bands centered in 10.8 and $12 \mu \mathrm{m}$, phase, emissivity, cloud temperature, etc.) the BTD can be related with the height and type of cloud. Considering these MODIS products in the same scene selected in the MSG image a $\mathrm{BTD}=1.1$ has been set to distinguish between low and high clouds.

Summarizing, the SWA-RTE procedure consists of:

(a) Applying the BTD threshold to discriminate low and high clouds;

(b) Applying the SWA to pixels with BTDs lower than 1.1 to retrieve the cloud temperature of low clouds;

(c) Applying the RTE-based procedure to pixels with BTDs higher than 1.1 to retrieve the emissivity and the cloud temperature;

(d) Estimating the cloud height from the cloud temperature using the vertical profiles of temperature in the scene.

\subsubsection{SWA-RTE Procedure Validation}

The MSG information does not include emissivity, phase and cloud temperature data. For this reason, to check the capabilities of the procedure, MODIS images have been used again. The procedure is equivalent although the coefficients of the SWA and the RTE approximation change because of the difference of the band widths between MODIS and MSG. In Fig.2 MODIS products are shown (cloud temperature (a), emissivity (b) and phase (c)). Analyzing these figures it is not difficult to see that the scene is dominated by low and hot clouds. Then some high clouds appear in the lower left corner and the upper part of the image. In Fig.3 the difference between the cloud temperature given by MODIS and the one calculated with the SWA (a), the RTE approximation (b) and SWA-RTE procedure (c) are shown. As it was expected, the SWA retrieves the cloud temperature with good accuracy whilst the RTE improves the results in high clouds. Nonetheless, there are some clouds whose temperature is not correctly determined. To work out the origin of these differences the RTE procedure has been analyzed in more detail and the emissivity calculated by the RTE method has been compared with the one of MODIS. In Fig.4 the difference between the emissivity calculated by the RTE approximation and the emissivity provided by MODIS is shown. Those differences are low (between -0.1 and 0.2) for high clouds, where the approximation is applicable. Therefore, the problem arises from the cloud temperature retrieval. To check if the small emissivity error can affect notably the cloud temperature retrieval, the RTE approximation has been applied using the MODIS emissivity instead of the emissivity calculated by the RTE approximation. The differences between the cloud temperature provided by MODIS and the ones retrieved with the RTE approximation (with MODIS emissivity) are shown in Fig.5. 


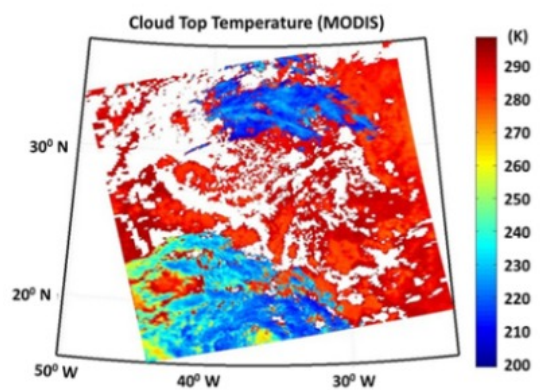

(a)

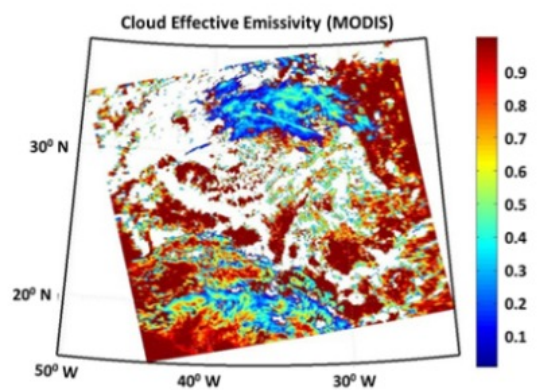

(b)

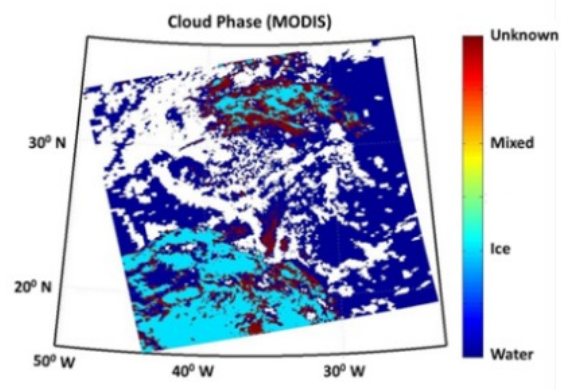

(c)

Figure 2: MODIS products: Cloud Top Temperature (a), cloud emissivity (b) and cloud phase (c).

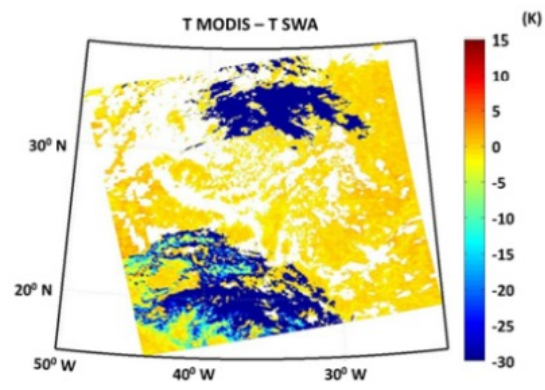

(a)

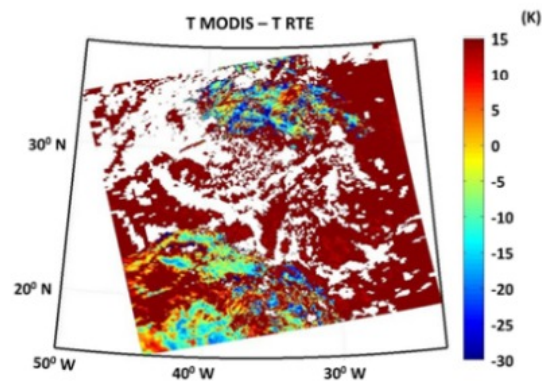

(b)

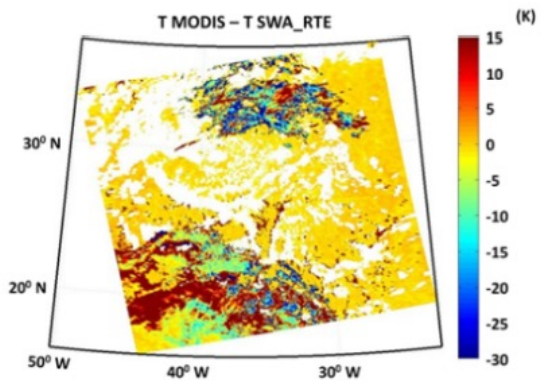

(c)

Figure 3: Differences between the cloud temperature provided by MODIS and the temperatures retrieved by the SWA (a), RTE approximation (b) and SWA-RTE procedure (c)

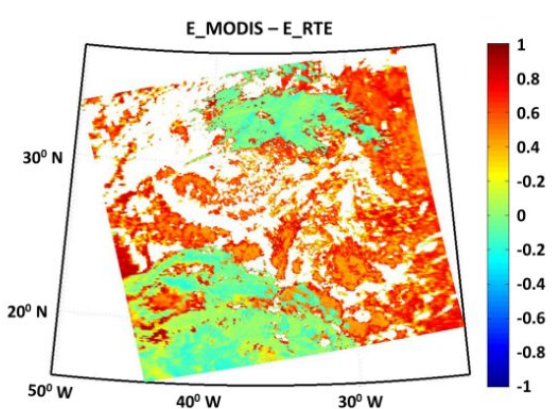

Figure 4: Emissivity difference (MODIS - RTE approx.)

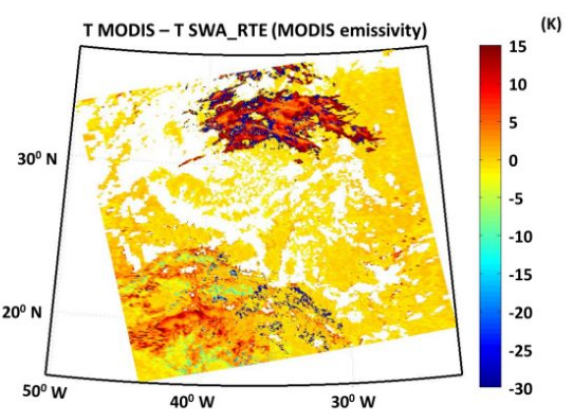

Figure 5: Cloud temperature difference (between MODIS and the RTE approximation) but using MODIS emissivity. 
The comparison between images Fig.3 (c) and Fig.5 reveals that the result of the high clouds located at the lower left corner improves when the MODIS emissivity is used although the results of the high clouds at the upper part of the images are not good enough yet. These facts demonstrate that although small emissivity errors can affect significantly the cloud temperature retrieval, there are other effects that influence the accuracy of the cloud temperature retrieval. Those effects can be related to cloud superposition or to the cloud structure near the top..$^{9,10}$

Nevertheless to avoid the effect of the emissivity errors in the final results and compare the methods themselves, the MODIS emissivity will be used in the RTE method to retrieve the cloud temperature.

After the cloud temperature is retrieved the cloud top height can be calculated from vertical profiles of temperature. This information is derived by interpolation of the profiles given by WRF. The procedure and its capabilities have been explained previously in. ${ }^{11}$

\section{DATA}

The validation of the three CTH retrieval methodologies presented in section 2 has been examined on a real case. The cloudy scene here considered occured the $8^{\text {th }}$ January 2015 in the centre of the Atlantic, in a huge zone located between 18-40 degrees in the SN direction and between 20-50 degrees in the WE direction.

Many reasons have brought to choose this scenario. Firstly, the selected area has been scanned quasi-synchronously by Meteosat 9 (at 15.44 UTC), Meteosat 10 (at 15.42 UTC) and Aqua-MODIS (at 15.40 UTC). This requirement is fundamental for our study as it allows the application of the stereo vision methodology and in the same time allows a direct comparison of the resulting CTHs with the CTH provided by the very accurate sensor MODIS, the spectroradiometer built on Aqua. As mentioned in the paragraph 2.3.2, as Meteosat does not provide complex cloud products (CTH, CTT, emissivity,...) for this scene, another reliable instrument had to be considered in order to check the validity of the procedures. In this work the MODIS Collection 6 data have been taken into account. Secondly, the cloudy case presents a very interesting weather scenario as it includes both low water clouds, where the SWA can be rightfully applied, and very high thin clouds, on which the stereo and the RTE methodologies can be investigated. Thirdly, the area close to the tropical zone allows to mantain the original nadir resolutions of the geostationary satellites being also completely scanned by Meteosat 9, which provides images above $15^{\circ} \mathrm{N}$.

\subsection{Meteorological observations}

From weather maps, MODIS Products and the lidar CALIPSO observations, a brief meteorological description of the analyzed scene can be done. A large subtropical centre of High Pressure is observed in the centre of Atlantic, probably the Azores High that tipically moves south of the Azores isles in winters. This huge anticyclonic area is delimited by a stationary front associated to a Low Pressure situated in the NW Atlantic. This front however does not involve the subtropical area here investigated. From MODIS products it is possible to recognize broken and scattered low-level clouds in the centre of the scene, while very few middle-level clouds $(2-7 \mathrm{~km})$ are present. Systems of thin ice clouds (cirrus) overlying lower-level water clouds $(<2 \mathrm{~km})$ are found in the N-centre and in SW areas of the scene.

\subsection{WRF simulation settings}

As mentioned before the WRF model Version 3.6 was used in this study. A simulation on the area of interest was done for the $8^{\text {th }}$ January 2015. The model was initialized by the ECMWF global model outputs released at $0.125^{\circ} \times 0.125^{\circ}$ of resolution. The spatial domain, centered in $27.5^{\circ} \mathrm{N}$ and $34.6^{\circ} \mathrm{W}$, was set with a resolution of $9 \mathrm{~km} \times 9 \mathrm{~km} \times 40$ vertical levels. The simulation started the day $8^{\text {th }}$ January 2015 at 00 UTC, setting the output time interval to 10 minutes, in order to focus the analysis at 15.40 UTC.

The main parametrizations were set to: Morrison 2-Moment scheme for mycrophysics, YSU scheme for planetary boundary layer, the Dudhia scheme for Short Wave and RRTM scheme for Long Wave Radiation, while the explicit choice has been set for the cumulus parametrization. 

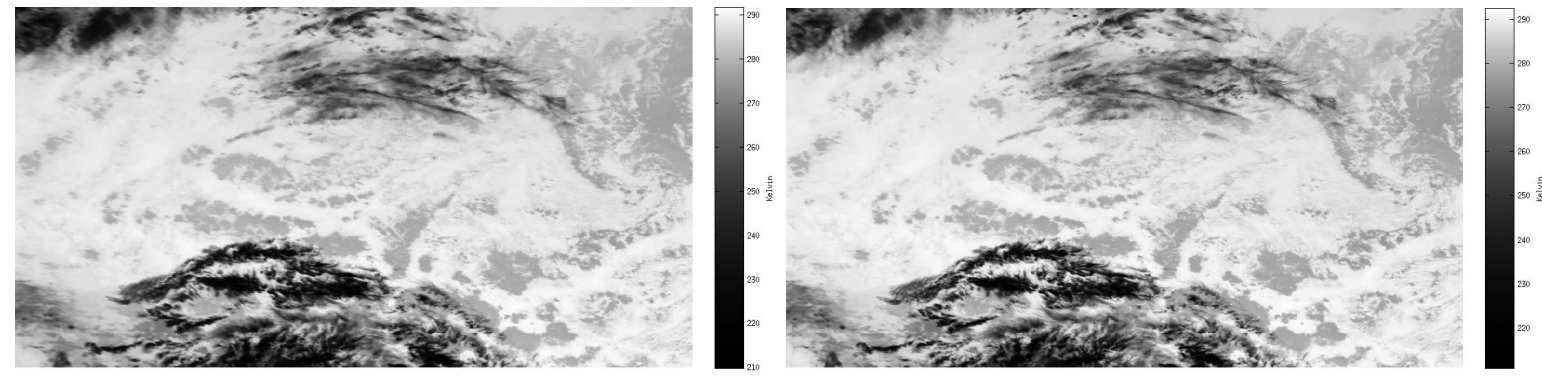

Figure 6: MSG stereo pair for band 10.8: MSG-2 image (left), and MSG-3 image (right)

\section{RESULTS}

\subsection{Stereo Vision Results}

The stereo algorithm has been tested on the MSG scene of $8^{\text {th }}$ January 2015 represented by the MSG-2 image at h:15:44 UTC and the MSG-3 at h:15:42 UTC, on the same Channel-9 and compared with the results obtained by MODIS. A few words on the stereo configuration are necessary to understand better the quality of the results. The acquisition time reported above regards the beginning of the scanning process, hence observing that the MSG-2 scans only the North Hemisphere, while MSG-3 starts from the South to the North Hemisphere, the region selected in this work is acquired by both satellites with a time lag of around 8 minutes. This can be a cause of problems as the stereo reconstruction method requires that the scene is static, that is, the two images are taken almost simultaneously. A time delay of about 8 minutes between the two acquisitions can sometimes easily break the static scene constraint, because of the natural not-rigid motion of the clouds, if extra information, such as wind speed and direction, are not taken into account. In this work we only use the information obtained from the IR images.

Being this approach independent from the meaning of the image values, for this experiment we used both the original radiance data and the images obtained transforming these data in BT. Since we did not notice relevant differences in the quality of the results among these different stereo pairs, here we report only the reconstruction obtained from the BT.

The stereo pair used in this experiment is shown in Fig.6. In Fig.7 we show the CTH obtained by our algorithm, and the disparity map obtained from the stereo matching procedure from which the heights are computed. The disparities recovered are very small, no more than two pixels, and since the disparity is quantized, even a small error (say half a pixel) account for a $25 \%$ error.

The height map obtained from MODIS that is used as ground truth for the comparison is shown in Fig.8, together with the distribution of the heights in the scene considered. From the histogram it is clear that in almost $85 \%$ of the scene the height is below $8 \mathrm{Km}$, meaning that, as discussed in Section (2.1), for the largest part of the scene the height cannot be measured using this method.

A quantitative analysis of the result is shown in Fig.9. From the histogram it is clear that almost $65 \%$ of the error for reconstructed height is less than $3 \mathrm{Km}$, that is, according to (1), well below half a pixel error in the disparity. For almost $75 \%$ of the pixels we have an error within $8 \mathrm{Km}$ that accounts for the large part of low clouds for which the height is overall estimated at $0 \mathrm{Km}$. Some parts of the higher clouds are missed by the stereo matching, most probably because of a non-rigid transformation of the scene due to the large time delay between the acquisition of the two images.

\subsection{WRF Cloud Fraction Results}

The WRF Cloud Fraction output of simulated at 15.40 UTC has been analyzed. The threshold was set to 0.9, that means that each model grid-box with a fraction of cloudiness higher than this is cloud. Fig.10 shows the difference in height between the MODIS product, used as reference, and the CTH retrieved from the WRF Cloud Fraction method. Fig.11 reports the histogram of the same height discrepancies. The errors in height for low water clouds are quite low, as the InterQuartileRange is $1.25 \mathrm{~km}$ and the median $-0.5 \mathrm{~km}$. However the 

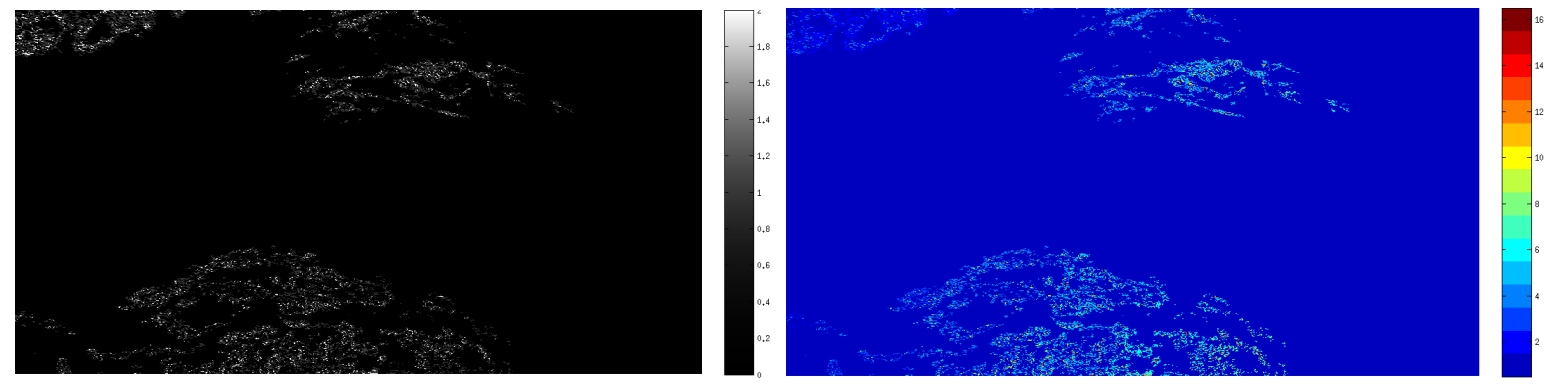

Figure 7: Disparity obtained by the stereo matching procedure (left), and CTH recovered by the stereo algorithm (right).
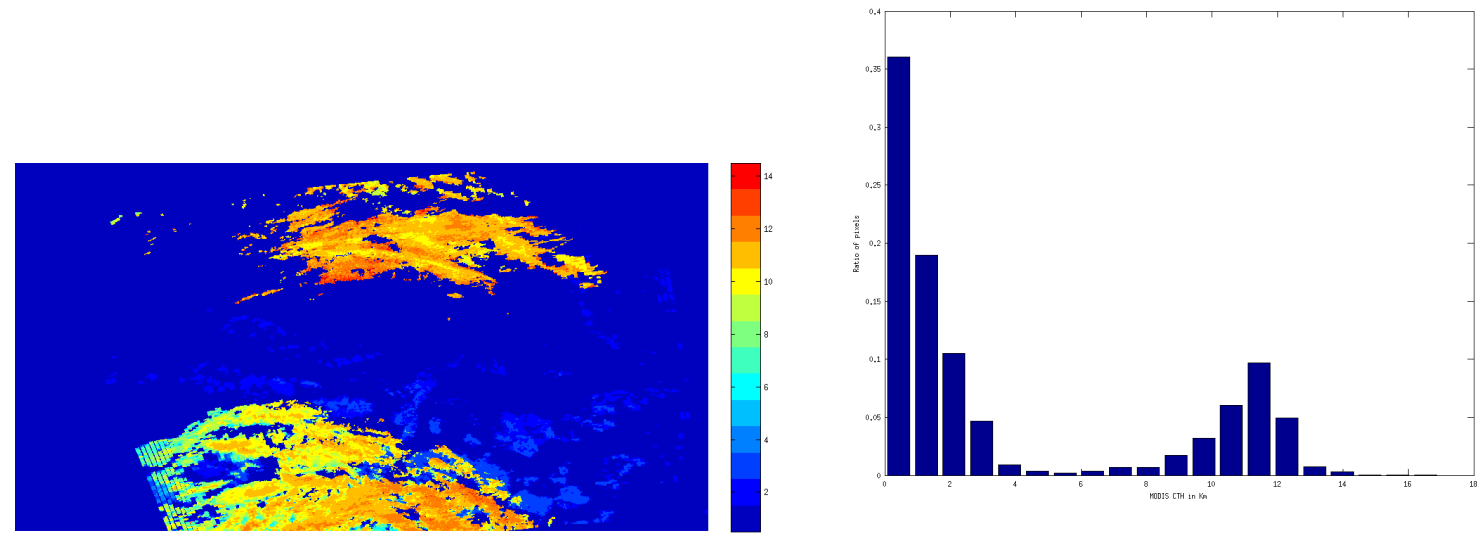

Figure 8: Heights obtained from MODIS and used as ground truth (left), and corresponding distribution of the heights (right).
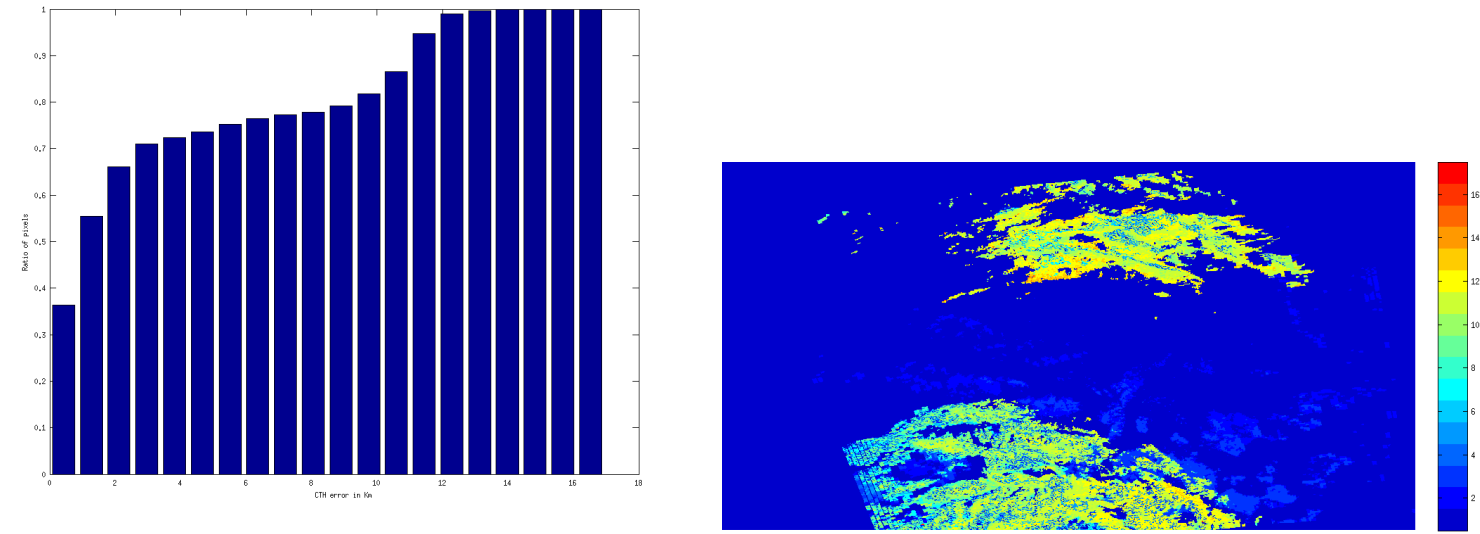

Figure 9: MSG: Cumulative histogram of the error of the recovered height map with respect to the MODIS height map (left); difference between the two height maps (right). 


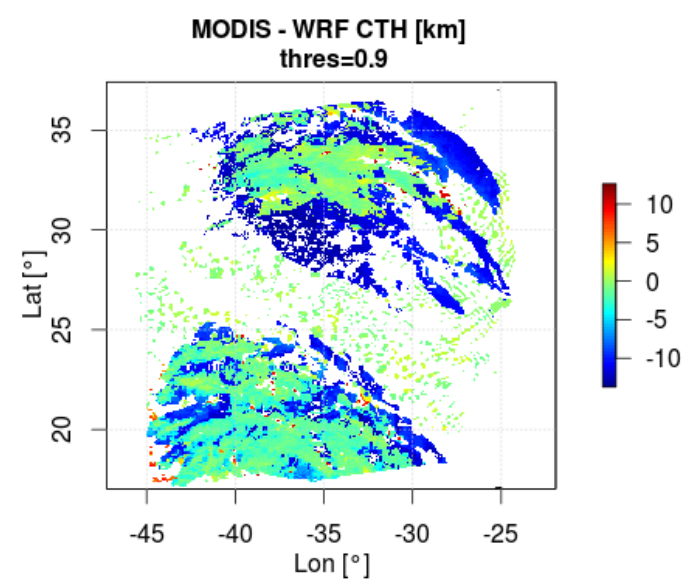

Figure 10: Error between MODIS CTH and the height retrieved from the WRF Cloud Fraction method [km].

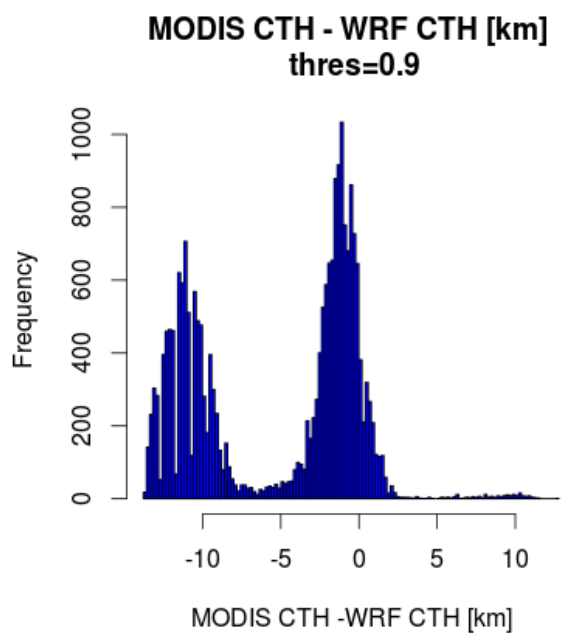

Figure 11: Histogram of the error between MODIS $\mathrm{CTH}$ and the height retrieved from the WRF Cloud Fraction method [km].

model detects just a few part of the low convective clouds that cover the ocean surface and seen by MODIS. The contrary is found for high $(7-12 \mathrm{~km})$ and very high clouds $(>12 \mathrm{~km})$. The large high cloud in the SW of the image is well reproduced, while the cloudy system in the North-Centre of the scene, probably made of multilayer clouds with ice very thin-cirrus-clouds on the top (as seen from MODIS products), is overestimated. That error makes the IQR for all the high clouds presented in the scene rising in value up to $9.5 \mathrm{~km}$ and the median up to $-3.6 \mathrm{~km}$, even though the height retrieval results for the huge cloud system in the SW are good.

\subsection{Radiative Transfer Method Results}

The procedure explained in Section 2.3.2 has been applied to the MSG images corresponding to the scene described previously. The surface temperature required for the cloud temperature retrieval has been obtained from MODIS products. For this reason the area of the final result corresponds to the MODIS image. In Fig.12 the height retrieved by the radiative transfer method is depicted. This result shows that low and mid-level clouds are predominant in the scene although some high clouds appear in the lower left corner and in the upper central zone. To check this result these heights have been compared to the heights given by MODIS. The difference is shown in Fig. 13 which shows that the discrepancies are very low, especially for low and mid-level clouds, where the SWA is applied. However there are few pixels showing higher differences. These pixels correspond mainly to transition borders between the cloud and clear sky where pixels not completely covered by the cloud are probable.

Nevertheless if the images in Fig.12 and in Fig.13 are compared with images of Section 2.3.2 it can be noticed that some pixels have disappeared, especially in the area of the upper high clouds. Looking into the result matrix we have detected that those pixels are marked as Not a Number (NaN). To investigate the source of this failure some intermediate results have been analyzed. In Fig.14 the cloud temperature retrieval is shown. The temperatures depicted in the figure are congruent with the heights and the temperatures given by MODIS but the image presents again the same problem: some pixels are classified as NaN. To work out if the failure is due to the procedure or the data inputs the MSG and MODIS radiances corresponding to the band centered in 10.8 have been compared. In Fig.15 the comparison between both radiances is presented. The difference is negligible in most part of the image, however there are some pixels where the discrepancies are appreciable reaching values of $\pm 60 \mathrm{~mW} / \mathrm{m}^{2} \mathrm{Sr} \mathrm{cm}^{-1}$. Precisely these pixels correspond to pixels marked as NAN in the height result image. The discrepancies can be related to advection movements of the height clouds which can be appreciable considering the small differences in the acquisition time or also they can be due to the different resolution of MSG 


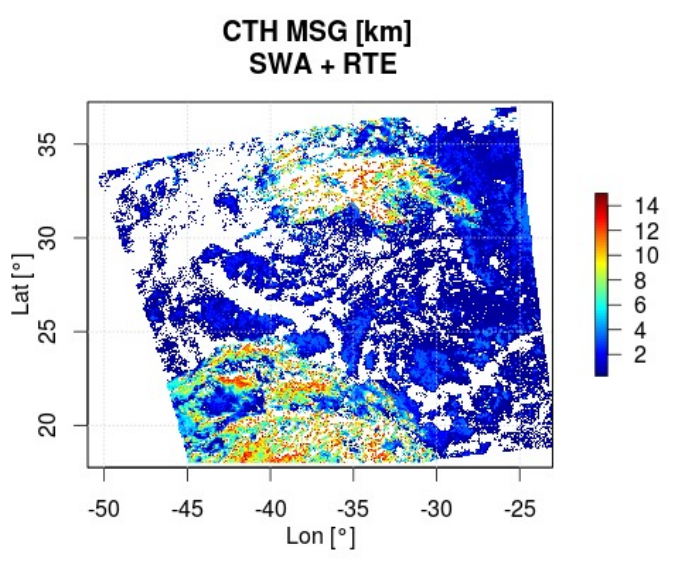

Figure 12: Cloud height retrieved by the radiative transfer method and 9 and 10 MSG channels.

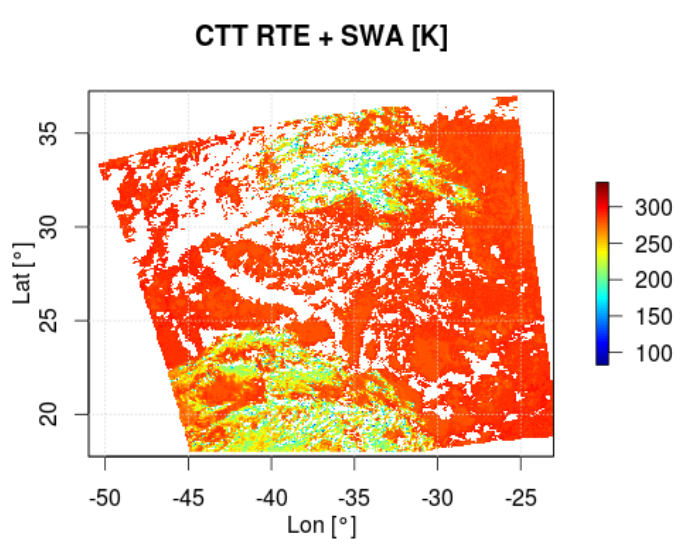

Figure 14: Temperature retrieved by the radiative transfer method from MSG images in channels 9 and 10 .

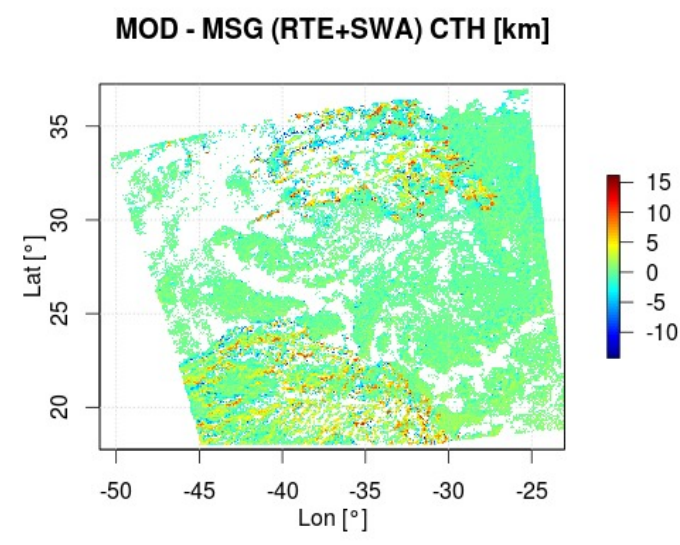

Figure 13: Difference between the height provided by MODIS and the height retrieved by the radiative transfer method.

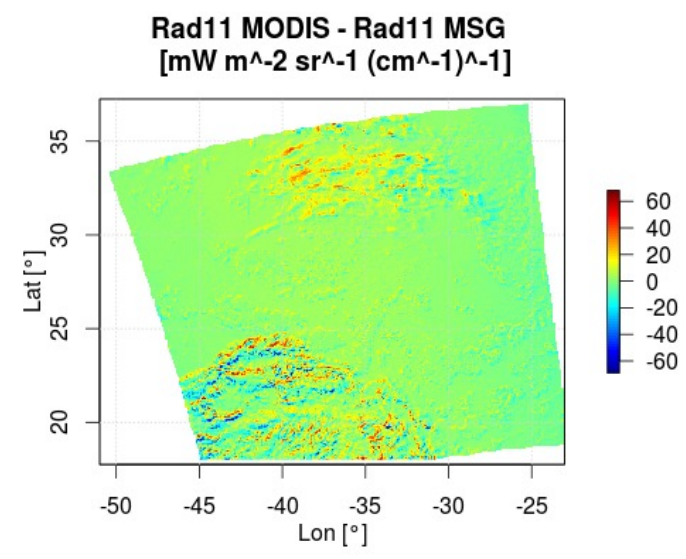

Figure 15: Discrepancies between the radiance measured by MSG (channel 9) and MODIS (31 band) 
and MODIS. In any case, this problem should be investigated in the future. Nevertheless, considering the small number of pixels affected by this issue, the applicability of the radiative transfer method is still valid and the results can be used in the general purpose of this work.

\section{CONCLUSION}

In this work three different methodologies of cloud height retrieval have been applied to an MSG scene. The application of Stereo Vision to MSG images provides good results in high level clouds, less than $3 \mathrm{~km}$ in the $65 \%$ of the pixels, although it is limited by the baseline between the stereo pair (MSG-2 and MSG-3) and consequently the low level clouds are not well determined. In addition the cloud motion might be one of the reasons of discrepancies in the retrieved heights, where the comparison between radiances of MODIS and MSG reveals variations in the high level clouds.

The WRF cloud fraction method is able to recognize the cloudy systems detected by MODIS sensor. The height of low water clouds is well retrieved, although their cloud cover is underestimated by the model. The method works well for high clouds associated to large scale events (as the ones located in the SW of the scene), while its performance gets worst for very-high thin clouds. The cloud cover of this type of systems, like the ones detected in the North-Centre of the scene, is overestimated. However the height of the model grid-boxes properly recognized as very-high thin clouds is quite well retrieved.

Finally, the radiative transfer method provides very accurate heights for low level clouds, although the height of high clouds is affected by the sensitivity of the RTE retrieval algorithm to emissivity errors and other effects related to the radiative physical process itself. In fact it makes difficult to determine the CTH since the level where the radiation comes is not well known as it depends on the cloud structure.

These results allow us to conclude that the combination of the three methodologies is a good alternative to provide very accurate heights for all level clouds when applied to MSG-2 and MSG-3 satellites. In addition, CTT and cloud emissivity are also supplied. All this information would be provided with high temporal resolution in a very extensive area, being highly valuable for meteorological research. Moreover, since the bands used in this work are provided by many instruments, the radiative transfer method can be applied to other data making easier the inter-comparison between different instruments. The use of IR bands also extends the coverage of application to night period.

\section{Acknowledgments}

The authors acknowledge the MODIS Science team for the Science Algorithms, the Processing Team for producing MODIS data, and the GES DAAC MODIS Data Support Team for making MODIS data available to the user community. We also thank EUMESAT for providing Meteosat data.

\section{REFERENCES}

[1] G. Seiz, S. Tjemkes, and P. Watts, "Multiview cloud-top height and wind retrieval with photogrammetric methods: Application to meteosat-8 HRV observations," Journal of Applied Meteorology and Climatology 46(8), pp. 1182-1195, 2007.

[2] J. D. Manizade, Kathrine F.and Spinhirne and R. S. Lancaster, "Stereo cloud heights from multispectral ir imagery via region-of-interest segmentation," IEEE T. Geoscience and Remote Sensing 44(9), pp. 24812491, 2006.

[3] W. Skamarock, J. Klemp, J. Dudhia, D. Gill, D. Barker, and M. Duda, "A description of the advanced research wrf version 3," tech. rep., NCAR, 2008.

[4] "Vapor," tech. rep., www.vapor.ucar.edu.

[5] T. Inoue, "On the temperature and effective emissivity determination of semi-transparent cirrus clouds by bispectral measurements in the 10 micron window region," Journal of Meteorology Society Japan 63, pp. 88-99, 1985.

[6] K. Heidinger and M. Pavolonis, "Gazing at cirrus clouds for 25 years through a split window. part i: Methodology," Journal of Applied Meterology and Climatology 48, pp. 1100-1116, 2014. 
[7] C. Wang, "A physically based algorithm for non-blackbody correction of cloud-top temperature and application to convection study," Journal of Applied Meteorology and Climatology 53, pp. 1844-1856, 2014.

[8] S. Briz, A. J. de Castro, I. Fernández-Gómez, I. Rodríguez, and F. López, "Remote sensing of water cloud temperature with an infrared camera on board the international space station in the frame of japan experiment module-extreme universe space observatory mission," Journal of Applied Remote Sensing 8, 2014.

[9] P. Minnis, C. R. Yost, S. Sun-Mack, and Y. Chen, "Estimating the top altitude of optically thick ice clouds from thermal infared satellite observations using calipso data," Geophysical Research Letters 35, 2008.

[10] E. E. Borbas, S. W. Seemann, A. Kern, L. Moy, J. Li, L. Gumley, and W. P. Menzel, "Modis atmospheric profile retrieval algorithm theoretical basis document," tech. rep., gfsc.nasa, 2011.

[11] D. Carli, C. Cassardo, M. Bertaina, S. Ferrarese, R. Cremonini, and I. Tabone, "The weather research and forecasting (wrf) model contribution to the atmospheric conditions estimation during the euso-balloon experiment," The 34th International Cosmic Ray Conference (ICRC) , 2015. 